\title{
Correlações genéticas entre escores visuais e características reprodutivas em bovinos Nelore usando inferência bayesiana
}

\author{
Arione Augusti Boligon(1) e Lucia Galvão de Albuquerque ${ }^{(2)}$
}

(1)Universidade Estadual Paulista, Faculdade de Ciências Agrárias e Veterinárias, Departamento de Zootecnia, Via de acesso Prof. Paulo Donato Castellane, s/nº, CEP 14884-900 Jaboticabal, SP. E-mail: arioneboligon@yahoo.com.br, Igalb@fcav.unesp.br

Resumo - $\mathrm{O}$ objetivo deste trabalho foi estimar a herdabilidade e as correlações genéticas entre escores visuais e características reprodutivas de animais da raça Nelore. As características avaliadas foram: precocidade, musculatura, e escores de conformação à desmama ( $\mathrm{PD}, \mathrm{MD}$ e $\mathrm{CD}$, respectivamente) e ao sobreano (PS, MS e CS, respectivamente); idade ao primeiro parto (IPP); e perímetro escrotal (PE). Foram utilizadas informações de 66.244 animais, nascidos entre 1990 e 2006. Os parâmetros genéticos foram estimados em análises bicaracterísticas, com inferência bayesiana. Foi utilizado um modelo linear para IPP e PE, e um modelo não linear ("threshold") para os escores visuais. As herdabilidades estimadas foram: $\mathrm{CD}, 0,19 \pm 0,02 ; \mathrm{PD}, 0,23 \pm 0,02 ; \mathrm{MD}, 0,20 \pm 0,02$; CS, $0,26 \pm 0,01$; PS, $0,33 \pm 0,02$; MS, $0,32 \pm 0,02$; IPP, $0,16 \pm 0,03$; e PE, $0,36 \pm 0,02$. As correlações genéticas estimadas entre os escores visuais e IPP foram negativas, de $-0,18 \pm 0,03$ a - $0,29 \pm 0,02$. Correlações genéticas positivas foram obtidas entre os escores visuais e o PE, de $0,19 \pm 0,01$ a $0,31 \pm 0,01$. A seleção de animais com os maiores escores visuais, principalmente ao sobreano, permite melhorar o desempenho reprodutivo dos rebanhos.

Termos para indexação: conformação, idade ao primeiro parto, musculatura, perímetro escrotal, precocidade.

\section{Genetic correlations between visual scores and reproductive traits in Nelore cattle using Bayesian inference}

\begin{abstract}
The aim of this work was to estimate the heritability and the genetic correlations between visual scores and reproductive traits of Nelore cattle. The traits evaluated were: finishing, muscling, and conformation scores at weaning (PD, MD, and CD, respectively) and yearling (PS, MS and CS, respectively); age at the first calving (IPP) and scrotal circumference (PE). The records of 66,244 animals born between 1990 and 2006 were used. The genetic parameters were estimated using bi-trait animal analyses with Bayesian inference. A linear model for IPP and PE and a nonlinear (threshold) model for visual scores were used. The heritability estimates were: CD, $0.19 \pm 0.02$; PD, $0.23 \pm 0.02$; MD, $0.20 \pm 0.02$; CS, $0.26 \pm 0.01$; PS, $0.33 \pm 0.02$; MS, $0.32 \pm 0.02$; IPP, $0.16 \pm 0.03$; and PE, $0.36 \pm 0.02$. Genetic correlation estimates between visual scores and IPP were negative, varying from $-0.18 \pm 0.03$ to $-0.29 \pm 0.02$. Positive genetic correlations were obtained between the visual scores and PE, from $0.19 \pm 0.01$ to $0.31 \pm 0.01$. Animal selection with the highest visual scores, mainly those taken after the yearling age, allows to improve herd reproductive traits.
\end{abstract}

Index terms: conformation, age at first calving, muscling, scrotal circumference, finishing.

\section{Introdução}

Em bovinos de corte, os escores visuais têm sido utilizados com o objetivo principal de identificar indivíduos com maior precocidade de terminação, melhor musculatura e conformação frigorífica, e sem tamanho excessivo na maturidade (Faria et al., 2009a; Weber et al., 2009). Trabalhos sobre a estimação dos coeficientes de herdabilidade demonstraram que os escores visuais são herdáveis (Koury Filho et al., 2009). A atribuição de escores de conformação, precocidade e musculatura pode ser realizada relativamente cedo na vida do animal e permite a avaliação de um grande número de animais, sem a necessidade de submetê-los a mensurações, o que torna o processo mais ágil e de menor custo.

As características reprodutivas são fundamentais para o melhoramento genético de bovinos de corte, pois podem limitar a intensidade de seleção, interferir no intervalo entre gerações, e são economicamente relacionadas ao sistema de produção. Entre as características indicadoras de fertilidade e precocidade sexual, a única amplamente empregada nos programas

Pesq. agropec. bras., Brasília, v.45, n.12, p.1412-1418, dez. 2010 
de melhoramento é o perímetro escrotal. $\mathrm{Na}$ raça Nelore, as estimativas de herdabilidade para o perímetro escrotal variam de 0,25 a 0,53 (Boligon et al., 2007; Yokoo et al., 2007).

Quando se consideram as características medidas em fêmeas, a idade ao primeiro parto é a mais utilizada para avaliar a fertilidade, pois é observada relativamente cedo, pode ser facilmente obtida e é expressa em grande parte das fêmeas colocadas em reprodução. Entretanto, selecionar diretamente as fêmeas quanto à menor idade ao primeiro parto não é simples, pois as características reprodutivas geralmente têm baixa herdabilidade, entre 0,14 e 0,19 (Pereira et al., 2002; Boligon et al., 2007). Além disso, alguns produtores atrasam a entrada de fêmeas na reprodução ao determinarem idade ou peso para início da vida reprodutiva, o que dificulta a identificação das fêmeas sexualmente precoces.

Para a elaboração de índices de seleção em bovinos de corte, o conhecimento de correlações genéticas entre as características de interesse econômico é fundamental. Nesse sentido, há necessidade de se verificar se as características indicadoras de fertilidade e precocidade sexual estão associadas aos escores visuais que são atualmente considerados nos programas de seleção.

Estudos que relacionam escores visuais com o desempenho reprodutivo em animais zebuínos são escassos na literatura, como foi verificado por Faria et al. (2009b), que estimaram as correlações genéticas entre os escores de conformação, estrutura física e musculatura com a idade ao primeiro parto e o perímetro escrotal, em bovinos da raça Nelore.

O objetivo deste trabalho foi estimar a herdabilidade e as correlações genéticas entre os escores visuais e características indicadoras de fertilidade e eficiência reprodutiva de animais da raça Nelore, por meio da inferência bayesiana.

\section{Material e Métodos}

Foram utilizados dados de 66.244 animais (machos e fềmeas), nascidos entre 1990 e 2006, pertencentes à Agropecuária Jacarezinho Ltda., fazenda localizada em Valparaíso, SP. Foram estudadas as características categóricas de conformação (CD), precocidade (PD) e musculatura (MD), obtidas à desmama e ao sobreano (CS, PS e MS, respectivamente) e, as características contínuas idade ao primeiro parto (IPP) e perímetro escrotal (PE).
Os animais foram pesados ao nascimento, à desmama (205 dias de idade) e ao sobreano (550 dias de idade). Tanto à desmama (de 140 a 270 dias de idade) como ao sobreano (de 400 a 600 dias de idade), os animais foram avaliados visualmente e receberam notas de um a cinco quanto à conformação, precocidade, musculatura e umbigo. Além disso, foi realizada a mensuração do perímetro escrotal, em animais com idade de 400 a 560 dias.

A seleção dos animais foi realizada com base em índices empíricos, compostos pelas características avaliadas à desmama e ao sobreano. Nesses dois períodos, os escores de conformação, precocidade e musculatura foram incluídos nos índices de seleção. Na desmama, descartaram-se $10 \%$ das fêmeas e $50 \%$ dos machos, e o restante da seleção foi realizada ao sobreano. O perímetro escrotal foi considerado somente na seleção realizada ao sobreano. Para estimar a quantidade de carne na carcaça do animal, o escore visual de conformação foi atribuído pelo comprimento e pela profundidade do corpo, associado ao desenvolvimento muscular e à harmonia geral do indivíduo. Com a avaliação do escore visual de precocidade, procurou-se estimar a capacidade do indivíduo em armazenar gordura, o que é indicação de rapidez para atingir o acabamento, enquanto o escore visual de musculatura é indicação do grau de desenvolvimento muscular apresentado pelo animal. Esses escores foram atribuídos a cada animal, relativamente à média do grupo de animais contemporâneos, e dependeram da concordância entre três avaliadores. Na Tabela 1, está apresentada a distribuição dos escores de conformação, precocidade e musculatura.

Tabela 1. Distribuição dos escores visuais de conformação, precocidade e musculatura em 66.244 bovinos (machos e fêmeas) da raça Nelore.

\begin{tabular}{lccccc}
\hline Característica $^{(1)}$ & \multicolumn{5}{c}{ Escore } \\
\cline { 2 - 6 } & 1 & 2 & 3 & 4 & 5 \\
\hline CD & 3.348 & 12.770 & 28.409 & 15.604 & 5.768 \\
PD & 3.173 & 10.321 & 30.432 & 16.687 & 5.286 \\
MD & 3.892 & 11.902 & 29.098 & 16.161 & 4.846 \\
CS & 2.980 & 9.098 & 17.982 & 8.752 & 3.261 \\
PS & 2.294 & 10.095 & 16.980 & 8.723 & 3.981 \\
MS & 3.754 & 9.540 & 16.032 & 9.813 & 2.934 \\
\hline
\end{tabular}

${ }^{(1)}$ Escore de conformação (CD), precocidade (PD) e musculatura (MD) à desmama; escores de conformação (CS), precocidade (PS) e musculatura (MS) ao sobreano. 
Para os escores obtidos à desmama, a definição do grupo de contemporâneos (GC) incluiu as variáveis sexo, fazenda da desmama, ano e estação de nascimento, e grupo de manejo ao nascimento e à desmama. Nos escores atribuídos ao sobreano, a fazenda e o grupo de manejo também foram incluídos no GC. $\mathrm{Na}$ IPP, o GC foi composto por fazenda da desmama e do sobreano, ano e estação de nascimento, grupo de manejo à desmama e ao sobreano, e tipo de serviço (inseminação artificial ou monta natural). No PE, o GC foi o mesmo utilizado para os escores visuais ao sobreano, porém sem o efeito de sexo.

Nos escores visuais, foram eliminados os GC com menos de oito animais. Para as características contínuas (IPP e PE), foram excluídos GC com menos de quatro animais e medidas de 3,5 desvios-padrão acima ou abaixo da média do seu GC. A estrutura geral do arquivo de dados analisado está descrita na Tabela 2.

Os componentes de variância e covariância e os parâmetros genéticos foram estimados por meio de análises bayesianas bicaracterísticas, tendo-se considerado um modelo animal linear para IPP e PE e um modelo animal não linear ("threshold") para os escores visuais. A combinação das características no modelo threshold-linear foi feita com o programa computacional THRGIBBSF90 (Misztal et al., 2002), que permite o estudo de características contínuas e categóricas na mesma análise. As estimativas a posteriori foram obtidas com a utilização do aplicativo POSTGIBBSF90 (Misztal et al., 2002). O modelo completo pode ser representado em notação matricial como $y=X \beta+Z_{a} a+Z_{m} m+Z_{c} c+\varepsilon$, em que: y é o vetor dos caracteres observados; $\beta$ é o vetor dos efeitos fixos; a é o vetor dos efeitos genéticos aditivos diretos; $m$ é

Tabela 2. Número de observações, médias e número de grupo de contemporâneos para as características avaliadas.

\begin{tabular}{lccc}
\hline Característica $^{(1)}$ & Observações & Média \pm DP & Grupo de contemporâneos \\
\hline CD & 65.899 & $3,11 \pm 0,92$ & 520 \\
PD & 65.899 & $3,21 \pm 0,91$ & 520 \\
MD & 65.899 & $3,06 \pm 0,93$ & 520 \\
CS & 42.073 & $3,05 \pm 0,90$ & 601 \\
PS & 42.073 & $3,12 \pm 0,93$ & 601 \\
MS & 42.073 & $2,99 \pm 0,92$ & 601 \\
IPP (dias) & 26.012 & $1.026,43 \pm 81,44$ & 453 \\
PE (cm) & 17.032 & $26,04 \pm 3,02$ & 402 \\
\hline
\end{tabular}

${ }^{(1)}$ Escore de conformação $(\mathrm{CD})$, precocidade $(\mathrm{PD})$ e musculatura $(\mathrm{MD})$ à desmama; escores de conformação (CS), precocidade (PS) e musculatura (MS) ao sobreano; idade ao primeiro parto (IPP); perímetro escrotal (PE); desvio-padrão (DP). o vetor dos efeitos genéticos aditivos maternos; c é $o$ vetor do efeito de ambiente permanente materno; $\varepsilon$ é o vetor dos efeitos residuais; e $X, Z_{a}, Z_{m}$ e $Z_{c}$ são as matrizes de incidência relacionadas a $\beta, \mathrm{a}, \mathrm{m}, \mathrm{c}, \mathrm{y}$. Considerou-se que $\mathrm{E}[\mathrm{y}]=\mathrm{X} \beta, \operatorname{Var}(\mathrm{a})=\mathrm{A} \otimes \Sigma_{\mathrm{a}}, \operatorname{Var}(\mathrm{m})$ $=\mathrm{A} \otimes \Sigma_{\mathrm{m}}, \operatorname{Var}(\mathrm{c})=\mathrm{I}_{\mathrm{Nm}} \otimes \Sigma_{\mathrm{c}} \mathrm{e} \operatorname{Var}(\mathrm{e})=\mathrm{I}_{\mathrm{N}} \otimes \Sigma_{\mathrm{e}}$, em que: $\Sigma_{\mathrm{a}}$ é a matriz de covariâncias genéticas aditivas; $\Sigma_{\mathrm{m}}$ é a matriz de covariâncias genéticas aditivas maternas; $\Sigma_{\mathrm{c}}$ é a matriz de covariâncias de ambiente permanente materno; $\Sigma_{\mathrm{e}}$ é a matriz de covariâncias residuais; A é a matriz de parentesco; I é uma matriz identidade; $\mathrm{Nm}$ é o número de mães dos animais com registros; $\mathrm{N}$ é o número de animais com registros; e $\otimes$ denota o produto direto entre as matrizes. Considerou-se que os vetores a, m, c e $\varepsilon$ são independentes. Os efeitos genéticos maternos e de ambiente permanente materno foram limitados aos escores visuais obtidos à desmama (CD, PD e MD). O GC foi considerado como efeito sistemático e, como covariáveis, foram considerados os efeitos linear e quadrático da idade do animal à mensuração (exceto IPP) e a idade da vaca ao parto.

No modelo de limiar, considera-se que a escala subjacente apresenta distribuição normal contínua, representada como: $\mathrm{U} \mid \theta \sim \mathrm{N}\left(\mathrm{W} \theta, \mathrm{I} \sigma_{\mathrm{e}}{ }^{2}\right)$, em que: $\mathrm{U}$ é o vetor da escala base de ordem r; $\theta=\left(\beta^{\prime}, a^{\prime}, m^{\prime}, c^{\prime}\right)$ é o vetor dos parâmetros de locação de ordem $\mathrm{s}$, com $\beta$ definido sob o ponto de vista frequentista como efeitos fixos e ordem s, com a, m e c como efeitos aleatórios genéticos aditivos diretos, genéticos maternos e de ambiente permanente materno; W é a matriz de incidência conhecida, de ordem r por s; I é a matriz de identidade de ordem $r$ por $r$; e $\sigma_{\mathrm{e}}^{2}$ é a variância residual. Quando se considera que a variável na distribuição subjacente não é observável, a parametrização $\sigma_{\mathrm{e}}^{2}=1$ é geralmente adotada para que se possa identificá-la na função de verossimilhança (Gianola \& Sorensen, 2002). Tal pressuposição é padrão em análises para dados categóricos em modelo de limiar.

As características categóricas são determinadas por variáveis contínuas não observáveis, em escala subjacente, em que são fixados valores iniciais de limiares, tais que:

$$
\mathrm{t}_{1}<\mathrm{t}_{2} \mathrm{~K}<\mathrm{t}_{\mathrm{j}-1}, \text { com }_{0}=-\infty \text { e } \mathrm{t}_{\mathrm{j}}=\infty,
$$

em que jé o número de categorias. Os dados observáveis são dependentes da variável subjacente, que é limitada entre dois limiares não observáveis (Gianola \& Foulley, 1983). Assim, as categorias ou escores de $y_{i}$ 
(características categóricas), para cada animal i, são definidos por $\mathrm{U}_{\mathrm{i}}$, na escala subjacente:

$$
\mathrm{y}_{1}=\text { (1) } \mathrm{t}_{0}<\mathrm{U}_{\mathrm{i}} \leq \mathrm{t}_{1} ; \text {; (2) } \mathrm{t}_{1}<\mathrm{U}_{\mathrm{i}} \leq \mathrm{t}_{2} ;(3) \mathrm{t}_{2}<\mathrm{U}_{\mathrm{i}} \leq \mathrm{t}_{3} \text {; }
$$$$
\text { (4) } t_{3}<U_{i} \leq t_{4} ;(5) t_{4}<U_{i} \leq t_{5} \text {, para } i=1, \ldots, n \text {, }
$$

em que: $n$ é o número de observações. Após as especificações dos limiares $t_{0}$ a $t_{5}$, é necessário que um dos limiares (de $t_{1}$ a $t_{4}$ ) seja ajustado a uma constante arbitrária. Neste estudo, considerou-se $t_{1}=0$, tal que $o$ vetor de limiares estimáveis foi definido como $t=t_{2}$; $\mathrm{t}_{3}$ e $\mathrm{t}_{4}$.

Nas análises bicaracterísticas que contêm variáveis categóricas e contínuas, de acordo com o enfoque bayesiano, os vetores $\beta$, a, m e c são parâmetros de locação de uma distribuição condicional y $\mid \beta, \mathrm{a}, \mathrm{m}$, c. Foi considerado, a priori, que $\beta$ tem uma distribuição uniforme que reflete um conhecimento prévio vago sobre esse vetor. Aos demais componentes foram atribuídas distribuições Wishart. Assim, a distribuição de y, dados os parâmetros de locação e escala, foi considerada como:

$$
\mathrm{y} \mid \beta, \mathrm{a}, \mathrm{m}, \mathrm{c}, \mathrm{R} \sim \mathrm{N}\left[\mathrm{X} \beta+\mathrm{Z}_{\mathrm{a}} \mathrm{a}+\mathrm{Z}_{\mathrm{m}} \mathrm{m}+\mathrm{Z}_{\mathrm{c}} \mathrm{c}, \mathrm{I}_{\mathrm{N}} \mathrm{R}\right]
$$

(Cantet et al., 1992; Van Tassel \& Van Vleck, 1996).

Nas análises bicaracterísticas, foram originadas cadeias com comprimento de 500 mil ciclos, em que os primeiros 10 mil foram descartados. As amostras foram armazenadas a cada 10 ciclos. O período de descarte e o intervalo de amostragem foram estabelecidos empiricamente. A convergência foi verificada com a inspeção gráfica, valores amostrados x iterações, e com o critério proposto por Geweke (1992). Para todas as características, as médias das variâncias a posteriori, estimadas em cada uma das análises bicaracterísticas, foram usadas para a estimação da herdabilidade média a posteriori.

\section{Resultados e Discussão}

As herdabilidades diretas médias a posteriori, obtidas para os escores visuais mensurados à desmama, foram inferiores às obtidas ao sobreano (Tabela 3 ). Em geral, os escores visuais devem responder à seleção individual e, ao se considerar a mesma intensidade de seleção em ambas as idades (desmama e sobreano), a resposta à seleção para as avaliações visuais ao sobreano tende a ser superior.
Ao utilizar a inferência bayesiana em estudo de escores visuais de animais da raça Nelore, Faria et al. (2009a) relataram altos valores de herdabilidade para os escores de conformação, precocidade e musculatura à desmama $(0,68,0,65$ e 0,62 , respectivamente) e menores valores ao sobreano $(0,44,0,38$ e 0,32 , respectivamente). Cabe ressaltar que esses autores optaram por não considerar o efeito materno nas análises dos escores visuais à desmama, em razão da dificuldade de atingir a convergência da cadeia de Gibbs, quando são consideradas características "threshold" em análises com modelo animal.

Os valores de herdabilidade materna média, estimada para os escores visuais obtidos à desmama, foram: CD, $0,07 \pm 0,01$; PD, $0,06 \pm 0,01$; e MD, $0,06 \pm 0,01$. Em estudo sobre a raça Aberdeen Angus, Weber et al. (2009) relataram estimativas de herdabilidade materna superiores aos do presente trabalho. Apesar da pequena magnitude, o efeito genético materno não deve ser desprezado em análises de escores visuais à desmama, uma vez que, segundo Meyer (1992), o emprego de modelos que não consideram os efeitos maternos para características medidas à desmama pode resultar em maiores valores de variância genética

Tabela 3. Estatística descritiva das médias das estimativas de herdabilidade direta a posteriori, para as características estudadas, obtidas a partir de análises bayesianas bicaracterísticas.

\begin{tabular}{lcc}
\hline Estatística descritiva & Desmama & Sobreano \\
\hline Média \pm DP & $0,19 \pm 0,02$ & $0,26 \pm 0,01$ \\
Moda & 0,19 & 0,26 \\
Mediana & 0,19 & 0,26 \\
Região de credibilidade $(95 \%)$ & 0,16 a 0,22 & 0,23 a 0,30 \\
\hline & \multicolumn{2}{c}{ Precocidade } \\
Média \pm DP & $0,23 \pm 0,02$ & $0,33 \pm 0,02$ \\
Moda & 0,22 & 0,33 \\
Mediana & 0,23 & 0,32 \\
Região de credibilidade $(95 \%)$ & 0,18 a 0,25 & 0,29 a 0,35 \\
\hline & \multicolumn{2}{c}{ Musculatura } \\
Média \pm DP & $0,20 \pm 0,02$ & $0,32 \pm 0,02$ \\
Moda & 0,19 & 0,32 \\
Mediana & 0,20 & 0,32 \\
Região de credibilidade $(95 \%)$ & 0,17 a 0,23 & 0,29 a 0,35 \\
\hline & Idade ao primeiro parto Perímetro escrotal \\
Média \pm DP & $0,16 \pm 0,03$ & $0,36 \pm 0,02$ \\
Moda & 0,16 & 0,36 \\
Mediana & 0,16 & 0,36 \\
Região de credibilidade $(95 \%)$ & 0,12 a 0,19 & 0,33 a 0,42 \\
\hline
\end{tabular}

Pesq. agropec. bras., Brasília, v.45, n.12, p.1412-1418, dez. 2010 
aditiva direta e, consequentemente, em superestimação da herdabilidade quanto ao efeito direto.

A média a posteriori das estimativas de herdabilidade da característica IPP foi baixa $(0,16 \pm 0,03)$, e a estimativa do intervalo de $95 \%$ da maior densidade a posteriori variou de 0,12 a 0,19 , o que indica que a utilização dessa característica como critério de seleção deverá promover pequeno progresso genético quanto à precocidade sexual em rebanhos Nelore. Em um estudo dos diferentes modelos, em termos de efeitos fixos, para avaliar a idade ao primeiro parto de fêmeas da raça Nelore, Boligon et al. (2008) relataram valores de herdabilidade de 0,14 a 0,15. Esses autores salientaram que os diferentes modelos utilizados causaram pequenas diferenças nas estimativas de parâmetros genéticos dessa característica.

Segundo Silva et al. (2005), a seleção quanto à precocidade sexual de fêmeas Nelore deveria ser feita em animais mais jovens, pois quando as fêmeas são expostas antecipadamente à reprodução, há maior possibilidade de obtenção de um rápido ganho genético, uma vez que em animais com 16 meses de idade, a característica prenhez teve estimativa de herdabilidade $(0,52)$ maior do que nos animais com 24 meses de idade $(0,12)$.

A herdabilidade média estimada do perímetro escrotal foi moderada (Tabela 3), o que indica que parte substancial da variação nessa característica é determinada pela ação aditiva dos genes, portanto, o perímetro escrotal deve responder ao processo de seleção. A herdabilidade para esta característica encontra-se dentro dos limites relatados na literatura para a raça Nelore, que variam de 0,35 a 0,53 (Boligon et al., 2007; Yokoo et al., 2007; Boligon et al., 2010).

As correlações genéticas estimadas entre os escores visuais obtidos à desmama, ao sobreano e à idade ao primeiro parto foram negativas, tendo variado de baixa a média magnitude (Tabela 4). Observaram-se maiores associações genéticas entre a idade ao primeiro parto e os escores visuais avaliados ao sobreano, em relação aos escores visuais obtidos à desmama, provavelmente porque as características ao sobreano são obtidas mais próximo da idade ao primeiro parto (Faria et al., 2009b). No geral, pelos resultados do presente estudo, infere-se que a seleção para maiores valores de escores de conformação, precocidade e musculatura, principalmente ao sobreano, permite a obtenção de animais mais precoces sexualmente.
Em trabalho com animais Nelore, Faria et al. (2009b) relataram correlações genéticas favoráveis entre escores visuais de musculatura, estrutura física e conformação, obtidos aos 8,15 e 22 meses de idade, com a idade ao primeiro parto, e concluíram que a seleção por animais com melhor composição corporal pode levar à obtenção de animais mais precoces.

Ao utilizar dados de novilhas da raça Nelore, acasaladas próximo aos 18 meses de idade, Pita et al. (1998) e Semmelmann et al. (2001) relataram maiores escores visuais de conformação, precocidade e musculatura de novilhas prenhes, em relação às falhadas. Segundo esses autores, as estimativas de correlação fenotípica mostraram que os escores visuais ao sobreano podem auxiliar na indicação da idade ao primeiro parto de fêmeas Nelore.

As correlações genéticas médias a posteriori, entre os escores de avaliação visual e o perímetro escrotal, foram positivas (Tabela 4). Apesar de a seleção direta para o perímetro escrotal ser a forma mais eficiente de se obter melhorias na característica, pode-se observar que a seleção para os escores visuais à desmama e ao sobreano, além de permitir a seleção de animais com biótipos compatíveis com o sistema de produção, pode ser uma boa alternativa para melhorar a fertilidade e a precocidade sexual de rebanhos da raça Nelore.

Tabela 4. Estimativas a posteriori das correlações genéticas entre os escores visuais de conformação $(\mathrm{CD})$, precocidade (PD) e musculatura (MD) à desmama e escores de conformação (CS), precocidade (PS) e musculatura (MS) ao sobreano, e as características reprodutivas idade ao primeiro parto e perímetro escrotal, em animais da raça Nelore.

\begin{tabular}{lcccc}
\hline Características & Média \pm erro-padrão & Moda & Mediana & RC $(95 \%)^{(1)}$ \\
\hline \multicolumn{5}{c}{ Idade ao primeiro } \\
CD & $-0,21 \pm 0,02$ & $-0,21$ & $-0,22$ & $-0,34$ a $-0,05$ \\
PD & $-0,26 \pm 0,01$ & $-0,26$ & $-0,26$ & $-0,39$ a $-0,09$ \\
MD & $-0,18 \pm 0,03$ & $-0,18$ & $-0,18$ & $-0,33$ a $-0,01$ \\
CS & $-0,26 \pm 0,01$ & $-0,26$ & $-0,27$ & $-0,49$ a $-0,10$ \\
PS & $-0,29 \pm 0,02$ & $-0,29$ & $-0,29$ & $-0,56$ a $-0,11$ \\
MS & $-0,23 \pm 0,02$ & $-0,22$ & $-0,23$ & $-0,41$ a $-0,07$ \\
\hline & & Perímetro escrotal $(\mathrm{cm})$ & \\
CD & $0,19 \pm 0,01$ & 0,19 & 0,19 & $-0,08$ a 0,44 \\
PD & $0,24 \pm 0,02$ & 0,24 & 0,24 & $-0,10$ a 0,49 \\
MD & $0,21 \pm 0,02$ & 0,21 & 0,21 & 0,02 a 0,43 \\
CS & $0,27 \pm 0,01$ & 0,27 & 0,27 & 0,13 a 0,41 \\
PS & $0,31 \pm 0,01$ & 0,31 & 0,31 & 0,17 a 0,47 \\
MS & $0,29 \pm 0,02$ & 0,29 & 0,29 & 0,15 a 0,44 \\
\hline
\end{tabular}

${ }^{(1)} \mathrm{RC}$, região de credibilidade. 
Resultados semelhantes foram relatados por Faria et al. (2009b), que estimaram correlações genéticas de moderada magnitude entre os escores visuais e o perímetro escrotal mensurado ao sobreano. Segundo esses autores, as associações genéticas entre o perímetro escrotal, mensurado ao sobreano, e as características morfológicas, avaliadas aos 22 meses de idade, foram maiores em relação às obtidas entre as características morfológicas avaliadas em idades jovens (aos oito e 15 meses).

Na discussão do uso de escores visuais como critérios de seleção, as correlações genéticas entre estes e as características reprodutivas devem ser consideradas, uma vez que o desempenho reprodutivo dos animais é um dos fatores determinantes da eficiência total do sistema de produção de bovinos de corte. No rebanho estudado, os escores de conformação, precocidade e musculatura à desmama e ao sobreano e o perímetro escrotal são incluídas nos índices de seleção, o que pode ocasionar, a longo prazo, a redução da idade ao primeiro parto.

A inclusão de escores visuais de conformação, precocidade e musculatura nos índices de seleção poderá aumentar a frequência de genes desejáveis para a fertilidade e precocidade sexual. Cabe ressaltar que as estimativas de correlações obtidas no trabalho são escassas na literatura (Faria et al., 2009a), e que é importante a realização de trabalhos com diferentes conjuntos de dados e abordagens, para confirmar os resultados aqui obtidos.

\section{Conclusões}

1. Os escores visuais de conformação, precocidade e musculatura e o perímetro escrotal podem responder rapidamente à seleção individual.

2. A seleção quanto aos escores visuais deverá promover mudanças genéticas favoráveis à idade ao primeiro parto e ao perímetro escrotal de animais da raça Nelore.

\section{Referências}

BOLIGON, A.A.; RORATO, P.R.N.; ALBUQUERQUE, L.G. de. Correlações genéticas entre medidas de perímetro escrotal e características produtivas e reprodutivas de fêmeas da raça Nelore. Revista Brasileira de Zootecnia, v.36, p.565-571, 2007.

BOLIGON, A.A.; SILVA, J.A.V.; SESANA, R.C., SESANA, J.C.; JUNQUEIRA, J.B.; ALBUQUERQUE, L.G. Estimation of genetic parameters for body weights, scrotal circumference, and testicular volume measured at different ages in Nellore cattle. Journal of Animal Science, v.88, p.1215-1219, 2010.

BOLIGON, A.A.; VOZZI, P.A.; NOMELINI, J.; RORATO, P.R.N.; BEZERRA, L.A.F.; LÔBO, R.B. Parâmetros genéticos para idade ao primeiro parto estimados por diferentes modelos para rebanhos da raça Nelore. Ciência Rural, v.38, p.432-436, 2008.

CANTET, R.J.C.; FERNANDO, R.L.; GIANOLA, D. Bayesian inference about dispersion parameters of univariate mixed models with maternal effects: theoretical considerations. Genetics Selection Evolution, v.24, p.107-135, 1992.

FARIA, C.U. de; KOURY FILHO, W.; MAGNABOSCO, C.U.; ALBUQUERQUE, L.G. de; BEZERRA, L.A.F.; LÔBO, R.B. Bayesian inference in genetic parameter estimation of visual scores in Nellore beef-cattle. Genetics and Molecular Biology, v.32, p.753-760, 2009a.

FARIA, C.U. de; MAGNABOSCO, C.U.; ALBUQUERQUE, L.G. de; DE LOS REYES, A.; BEZERRA, L.A.F.; LÔBO, R.B. Análise bayesiana na estimação de correlações genéticas entre escores visuais e características reprodutivas de bovinos Nelore utilizando modelos linear-limiar. Arquivo Brasileiro de Medicina Veterinária e Zootecnia, v.61, p.949-958, 2009b.

GEWEKE, J. Evaluating the accurary of sampling-based approaches to the calculation of posterior moments. In: BERNARDO, J.M.; BERGER, J.O.; DAWID, A.P.; SMITH, A.F.M. Bayesian statistics 4. New York: Oxford University, 1992. p.625-631.

GIANOLA, D.; FOULLEY, J.L. Sire evaluation for ordered categorical data with a threshold model. Genetics Selection Evolution, v.15, p.201-224, 1983.

GIANOLA, D.; SORENSEN, D. Likelihood, Bayesian, and MCMC methods in quantitative genetics. New York: Springer-Verlag, 2002. 740p.

KOURY FILHO, W.; ALBUQUERQUE, L.G. de; ALENCAR, M.M. de; FORNI, S.; SILVA, J.A.V.; LOBO, R.B. Estimativas de herdabilidade e correlações para escores visuais, peso e altura ao sobreano em rebanhos da raça Nelore. Revista Brasileira de Zootecnia, v.38, p.2362-2367, 2009.

MEYER, K. Variance components due to direct and maternal effects for growth traits of Australian beef cattle. Livestock Production Science, v.31, p.179-204, 1992.

MISZTAL, I.; TSURUTA, S.; STRABEL, T.; AUVRAY, B.; DRUET, T.; LEE, D.H. BLUPF90 and related programs (BGF90). In: WORLD CONGRESS ON GENETICS APPLIED TO LIVESTOCK PRODUCTION, 7., 2002, Montpellier. Proceedings. Montpellier: INRA: CIRAD, 2002.

PEREIRA, E.; ELER, J.P.; FERRAZ, J.B.S. Análise genética de características reprodutivas na raça Nelore. Pesquisa Agropecuária Brasileira, v.37, p.703-708, 2002.

PITA, F.V.; FRIES, L.A.; ALBUQUERQUE, L.G. Utilização de escores visuais como indicadores da idade à maturidade sexual de novilhas Nelore. In: SIMPÓSIO NACIONAL DE MELHORAMENTO ANIMAL, 2., 1998, Uberaba. Anais. Viçosa: Sociedade Brasileira de Melhoramento Animal, 1998. p.383-384. 
SEMMELMANN, C.E.N.; LOBATO, J.F.P.; ROCHA, M.G. da. Efeito de sistemas de alimentação no ganho de peso e desempenho reprodutivo de novilhas Nelore acasaladas aos 17/18 meses. Revista Brasileira de Zootecnia, v.30, p.835-843, 2001.

SILVA, J.A. II de V.; DIAS, L.T.; ALBUQUERQUE, L.G. de. Estudo genético da precocidade sexual de novilhas em um rebanho Nelore. Revista Brasileira de Zootecnia, v.34, p.1568-1572, 2005.

VAN TASSEL, C.P.; VAN VLECK, L.D. Multiple-trait Gibbs sampler for animal models: flexible programs for Bayesian and likelihood-based (co)variance component inference. Journal of Animal Science, v.74, p.2586-2597, 1996.
WEBER, T.; RORATO, P.R.N.; LOPES, J.S.; COMIN, J.G.; DORNELLES, M. de A.; ARAÚJO, R.O. de. Parâmetros genéticos e tendências genéticas e fenotípicas para características produtivas e de conformação na fase pré-desmama em uma população da raça Aberdeen Angus. Revista Brasileira de Zootecnia, v.38,p.832-842, 2009.

YOKOO, M.J.I.; ALBUQUERQUE, L.G. de; LÔBO, R.B.; SAINZ, R.D.; CARNEIRO JÚNIOR, J.M.; BEZERRA, L.A.F.; ARAUJO, F.R. da C. Estimativas de parâmetros genéticos para altura do posterior, peso e circunferência escrotal em bovinos da raça Nelore. Revista Brasileira de Zootecnia, v.36, p.1761-1768, 2007.

Recebido em 12 de novembro de 2009 e aprovado em 30 de setembro de 2010 